\title{
Conversando sobre higiene com adolescentes escolares: um relato de experiência
}

\author{
Talking about hygiene with school adolescents: an experience report \\ Hablar de higiene con los adolescentes escolares: un informe de experiencia
}

Gabriela Oliveira Parentes da Costa ORCID: https://orcid.org/0000-0001-9473-8986 Instituto Federal do Maranhão, Brasil E-mail: gabiparents@hotmail.com

Isaura Danielli Borges de Sousa ORCID: https://orcid.org/0000-0001-7240-5072 Universidade Federal do Piauí, Brasil E-mail: isauradanielli@ufpi.edu.br

Ravena de Sousa Alencar Ferreira ORCID: https://orcid.org/0000-0001-7311-2212 Universidade Federal do Piauí, Brasil E-mail: ravenaa89@gmail.com

Naianne Geórgia Sousa de Oliveira ORCID: https://orcid.org/0000-0002-2949-0803 Faculdade Santa Luzia, Brasil

E-mail: naianne.enfermeira@gmail.com

Luciana Spindola Monteiro Toussaint ORCID: https://orcid.org/0000-0001-7691-1570 Fundação Municipal de Saúde, Brasil E-mail: lucianaspindola09@gmail.com Adalberto Fortes Rodrigues Júnior ORCID: https://orcid.org/0000-0002-5169-0027 Universidade Federal do Piauí, Brasil E-mail: jrfortes04@yahoo.com

Rebeca Natacha Barbosa Vieira ORCID: https://orcid.org/0000-0001-8306-5771 Faculdade Aliança, Brasil

E-mail: rebeca.natacha02@gmail.com

Érida Zoé Lustosa Furtado

ORCID: https://orcid.org/0000-0002-6162-7558 Universidade Federal do Piauí, Brasil

E-mail: eridazoe@hotmail.com

Maryanne Marques de Sousa

ORCID: https://orcid.org/0000-0001-9302-8499 Hospital Universitário da Universidade Federal do Piauí, Brasil

E-mail: maryannemarques8@ hotmail.com

Liana Regina Gomes de Sousa ORCID: https://orcid.org/0000-0002-9952-4204 Universidade Federal do Piauí, Brasil E-mail: liana.rgs@hotmail.com

Angélica de Oliveira Lino Araújo ORCID: https://orcid.org/0000-0002-6111-8470 Faculdade São Gabriel, Brasil

E-mail: angelica_lyno@hotmail.com

Luciane Resende da Silva Leonel ORCID: https://orcid.org/0000-0003-1787-1673

Universidade Estadual do Maranhão, Brasil

E-mail: lucianeresendelinda@gmail.com

Otília Maria Reis Sousa Tinel

ORCID: https://orcid.org/0000-0001-9306-7543

Universidade Federal do Piauí, Brasil

E-mail: otiliatinel1 @ outlook.com

Natália Freire Fontenele Gonzalez ORCID: https://orcid.org/0000-0003-1230-9905

Universidade Estadual do Maranhão, Brasil E-mail: freirefontenele@ hotmail.com

Karolinne Adrião de Oliveira

ORCID: https://orcid.org/0000-0002-9134-4292

Faculdade Maurício de Nassau, Brasil

E-mail: karoladriao_oliveira@hotmail.com 


\begin{abstract}
Resumo
Introdução: O ambiente escolar é um espaço pluridisciplinar e multidisciplinar, em que todos indivíduos corroboram para construção do conhecimento e saberes, sendo um local propício para se trabalhar ações que promoção a saúde e prevenção de doenças e agravos. Metodologia: Trata-se de um relato de experiência de uma atividade desenvolvida em 2019, com adolescentes na faixa etária de 14 anos, ingressantes no primeiro ano do ensino médio. A ação foi elaborada baseando-se no modelo de Ruas que consiste em um jogo educativo sobre higiene. Algumas questões foram adaptadas a realidade e à problemática da escola. O planejamento da ação, foi utilizada a metodologia da Teoria da Problematização, pautados nas ferramentas da educação popular e a troca de saberes, desenvolvidos por Paulo Freire. Relato de experiência e discussão: A idealização da intervenção surgiu pela necessidade de orientar alunos recém chegados ao ensino médio sobre bons hábitos de higiene. Professores queixavam-se da precariedade higiênica com que os discentes iam para a escola. A atividade ocorreu na forma de gincana, onde a turma foi dividida em duas equipes e as condutoras apresentaram uma série de perguntas sobre higiene, que tratavam sobre higiene bucal, higiene com os sapatos, com o fardamento escolar, com as axilas, e até mesmo com a região genital. Considerações Finais: A elaboração da intervenção teve o intuito de fortalecer o conhecimento sobre higiene entre os discentes, com compromisso e responsabilidade social, uma vez que o incentivo do autocuidado deve estar atrelado a autonomia e protagonismo dos sujeitos.
\end{abstract}

Palavras-chave: Higiene; Educação em saúde; Saúde escolar.

\begin{abstract}
Introduction: The school environment is a multidisciplinary and multidisciplinary space, in which all individuals contribute to the construction of knowledge and knowledge, being a favorable place to work on actions that promote health and prevent diseases and injuries. Methodology: This is an experience report of an activity developed in 2019 , with teenagers aged 14 years old, entering the first year of high school. The action was designed based on the Streets model, which consists of an educational game about hygiene. Some questions were adapted to the reality and problems of the school. The action planning used the Problematization Theory methodology, based on the tools of popular education and the exchange of knowledge, developed by Paulo Freire. Experience report and discussion: The idealization of the intervention arose from the need to guide students who had just arrived at high school on good hygiene habits. Teachers complained about the precarious hygiene with which students went to school. The activity took place in the form of a scavenger hunt, where the class was divided into two teams and the conductors presented a series of questions about hygiene, which dealt with oral hygiene, hygiene with shoes, school uniforms, armpits, and even with the genital region. Final Considerations: The intervention was designed to strengthen knowledge about hygiene among students, with commitment and social responsibility, since the encouragement of self-care must be linked to the autonomy and protagonism of the subjects.
\end{abstract}

Keywords: Hygiene; Health education; School health.

\title{
Resumen
}

Introducción: El entorno escolar es un espacio multidisciplinario y multidisciplinario, en el que todas las personas contribuyen a la construcción del conocimiento y el conocimiento, siendo un lugar propicio para trabajar en acciones que promuevan la salud y prevengan enfermedades y lesiones. Metodología: Se trata de un relato de experiencia de una actividad desarrollada en 2019, con adolescentes de 14 años, ingresando al primer año de secundaria. La acción se diseñó en base al modelo Streets, que consiste en un juego educativo sobre higiene. Algunas preguntas se adaptaron a la realidad y problemas de la escuela. La planificación de la acción utilizó la metodología de la Teoría de la Problematización, basada en las herramientas de la educación popular y el intercambio de conocimientos, desarrollada por Paulo Freire. Informe de experiencia y discusión: La idealización de la intervención surgió de la necesidad de orientar a los estudiantes recién llegados al bachillerato sobre buenos hábitos de higiene. Los profesores se quejaron de la precaria higiene con la que los alumnos iban a la escuela. La actividad se desarrolló en forma de búsqueda del tesoro, donde la clase se dividió en dos equipos y los conductores presentaron una serie de preguntas sobre higiene, las cuales versaron sobre higiene bucal, higiene con zapatos, uniformes escolares, axilas, e incluso con el región genital. Consideraciones finales: La intervención se diseñó para fortalecer los conocimientos sobre higiene entre los estudiantes, con compromiso y responsabilidad social, ya que el fomento del autocuidado debe estar ligado a la autonomía y protagonismo de los sujetos.

Palabras clave: Higiene; Educación para la salud; Salud escolar.

\section{Introdução}

O ambiente escolar é um espaço pluridisciplinar e multidisciplinar, em que todos indivíduos corroboram para construção do conhecimento e saberes, sendo um local propício para se trabalhar ações que promoção a saúde e prevenção de doenças e agravos (Cordeiro, 2019). 
Vários temas são trabalhados entre os adolescentes, principalmente a educação sexual, que é um dos assuntos mais difundidos nessa faixa etária. Porém, algumas problemáticas são deixadas de lado, como é o caso da higiene pessoal. Sendo negligenciadas, são desenvolvidas esporadicamente, não recebendo a devida importância no cotidiano (Mori \& Freitas, 2016; Terço \& Freitas, 2016).

A escola tem papel fundamental no processo educativo, sendo um ambiente propício para tratar de diversos assuntos e contribuir para a análise crítica do aluno (PIERI, 2020). Neste sentido, os conceitos de higiene devem trabalhados na escola de forma transversal, contribuindo para continuidade dentro e fora da sala de aula. Para que isso ocorra, a escola precisa implantar ações permanentes de educação em saúde, por meio de comissões que atuem orientando os adolescentes, seja no formato de palestras, rodas de conversas, gincanas, jogos ou quaisquer estratégias que incentivem o desenvolvimento de melhores hábitos de higiene (Cordellini, 2016; Cruz, 2018).

As estratégias usadas para encarar os problemas de saúde e transformar o comportamento de um grupo não devem se restringir apenas às doenças, mas devem abranger também as fragilidades relacionadas ao cuidado pessoal, que por sua vez, pode interferir no bem estar do indivíduo, afetando assim o modelo de saúde, que não visa somente a ausência de uma patologia e sim o bem-estar como um todo (Guerin et al. 2019; Ramos, 2020).

Contudo, assegurar o direito à saúde do adolescente é um grande desafio para os profissionais que atuam no ambiente escolar, uma vez que há necessidade de organização de horários junto à coordenação e docentes. Porém, a iniciativa de instituições de ensino, de levar informação em saúde é fundamental para prevenção de agravos e para o bem estar do indivíduo.

Quanto mais precoce for as ações de promoção em saúde, melhores serão as respostas. Contudo, deve-se atentar para a didática das atividades desenvolvidas, uma vez que a linguagem deve ser clara e que, para chamar a atenção dos adolescentes, algumas estratégias devem ser usadas (Silva, 2020; Mouta et al., 2020).

Neste sentido, o objetivo dessa pesquisa é relatar a vivência de uma intervenção anual sobre hábitos de higiene saudáveis entre adolescentes escolares.

\section{Metodologia}

Trata-se de um relato de experiência de profissionais de enfermagem atuantes em um Instituto Federal do Maranhão localizado em uma cidade pequena, pouco desenvolvida e com população registrando 49.000 habitantes em 2020.

O relato de experiência contribui para a disseminação do conhecimento a respeito de uma temática, de forma sistemática e cuidadosamente organizada, evidenciando o resultado da vivência dos pesquisadores, apoiadas em teorias e metodologias (Daltro \& Faria, 2019).

A atividade foi desenvolvida em 2019, com adolescentes na faixa etária de 14 anos, ingressantes no primeiro ano do ensino médio da referida instituição. Sendo estes os critérios de inclusão. Os critérios de exclusão foram alunos de outras turmas, que tivessem faltado a aula no dia da atividade ou estivessem de atestado médico.

A ação foi elaborada baseando-se no modelo de Ruas (2013) que consiste em um jogo educativo sobre higiene. Algumas questões foram adaptadas a realidade e à problemática da escola. Para a ação foi adotada uma proposta de gincana e contou com os questionamentos explicitados no quadro 1.

Para o planejamento da ação, foi utilizada a metodologia da Teoria da Problematização embasada na metodologia do Arco de Charles Maguerez (Figura 1), que tem como ponto de partida a realidade observada e identificação dos problemas existentes, determinação dos principais pontos a serem trabalhados, fundamentando-se em teorias, estratégias e hipóteses para a assim, intervir ou buscar soluções para o problema (Bordenave \& Pereira, 1989). Além disso, os fundamentos conceituais 
foram pautados nas ferramentas da educação popular e a troca de saberes, desenvolvidos por Paulo Freire, cuja finalidade é promover a autonomia e transformar a realidade de um grupo.

Figura 1: Etapas do Arco de Maguerez utilizado por Bordenave e Pereira (1989).

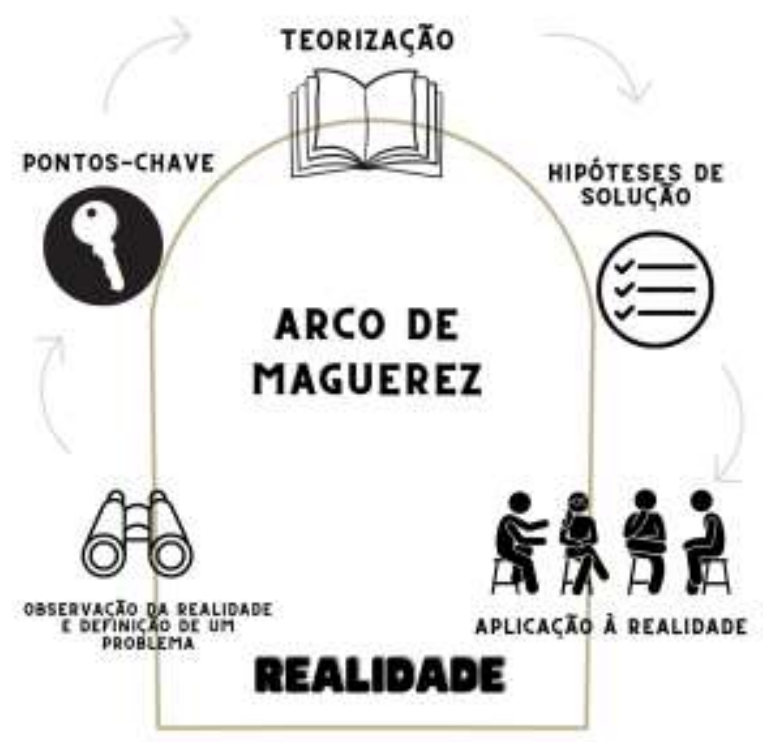

Fonte: Elaboração própria, baseada no modelo original.

A fundamentação teórica que embasou a ação e essa pesquisa, ocorreram nas bases científicas, Literatura LatinoAmericana e do Caribe em Ciências da Saúde e Scientific Electronic Library Online, de acordo com os descritores definidos nos Descritores em Ciências da Saúde (DeCS): Higiene; Educação em Saúde; Saúde Escolar.

\section{Relato da Experiência e Discussão}

A idealização da intervenção surgiu pela necessidade de orientar alunos recém chegados ao ensino médio sobre bons hábitos de higiene. Professores queixavam-se da precariedade higiênica com que os discentes iam para a escola. A partir disso, a equipe de enfermagem do local passou a organizar uma atividade anual sobre higiene corporal, visando a educação e conscientização dos alunos.

As ações ocorreram em todas as salas do primeiro ano, alternando datas e horários de acordo com a disponibilidade da agenda de disciplinas dos professores, que cederam o momento para a realização das atividades. A atividade ocorreu na forma de gincana, onde a turma foi dividida em duas equipes e as condutoras apresentaram uma série de perguntas sobre higiene, que tratavam sobre higiene bucal, higiene com os sapatos, com o fardamento escolar, com as axilas, e até mesmo com a região genital. As perguntas foram organizadas no Power Point e projetadas em sala. 
Quadro 1: Questões sobre higiene trabalhadas na gincana. Coelho Neto-MA, 2019.

\begin{tabular}{|l|l|}
\hline Tempo de escovação bucal adequado & Formas para prevenir "cecê" (bromidrose axilar) \\
\hline $\begin{array}{l}\text { Forma correta de escovação e utilização do fio } \\
\text { dental }\end{array}$ & $\begin{array}{l}\text { Cuidados com os pés para evitar "chulé" } \\
\text { (bromidrose plantar) }\end{array}$ \\
\hline Mau hálito & Cuidados com limpeza da região íntima \\
\hline Higienização das mãos e técnica correta & $\begin{array}{l}\text { Higienização das mãos antes de tocar nos } \\
\text { alimentos ou após o uso do banheiro }\end{array}$ \\
\hline Higiene das unhas e cabelos & Cuidados no banho \\
\hline $\begin{array}{l}\text { Higiene com as roupas de farda e roupas } \\
\text { íntimas }\end{array}$ & Cuidados durante o ciclo menstrual \\
\hline
\end{tabular}

Fonte: Autores.

Cada representante da equipe tinha um tempo para responder. A cada resposta correta, um quadrado marcado no chão era avançado e a cada erro, o representante do grupo oposto tinha a oportunidade de responder. Ao final, o representante da turma que chegou primeiro na linha de chegada levou um brinde para a equipe vencedora.

Algumas perguntas sobre higiene bucal não foram respondidas corretamente (Figura 2). Já, com relação a bromidrose axilar e podal, foi respondida com acerto (Figura 3).

Figura 2: Perguntas sobre higiene bucal. Coelho Neto-MA, 2019.
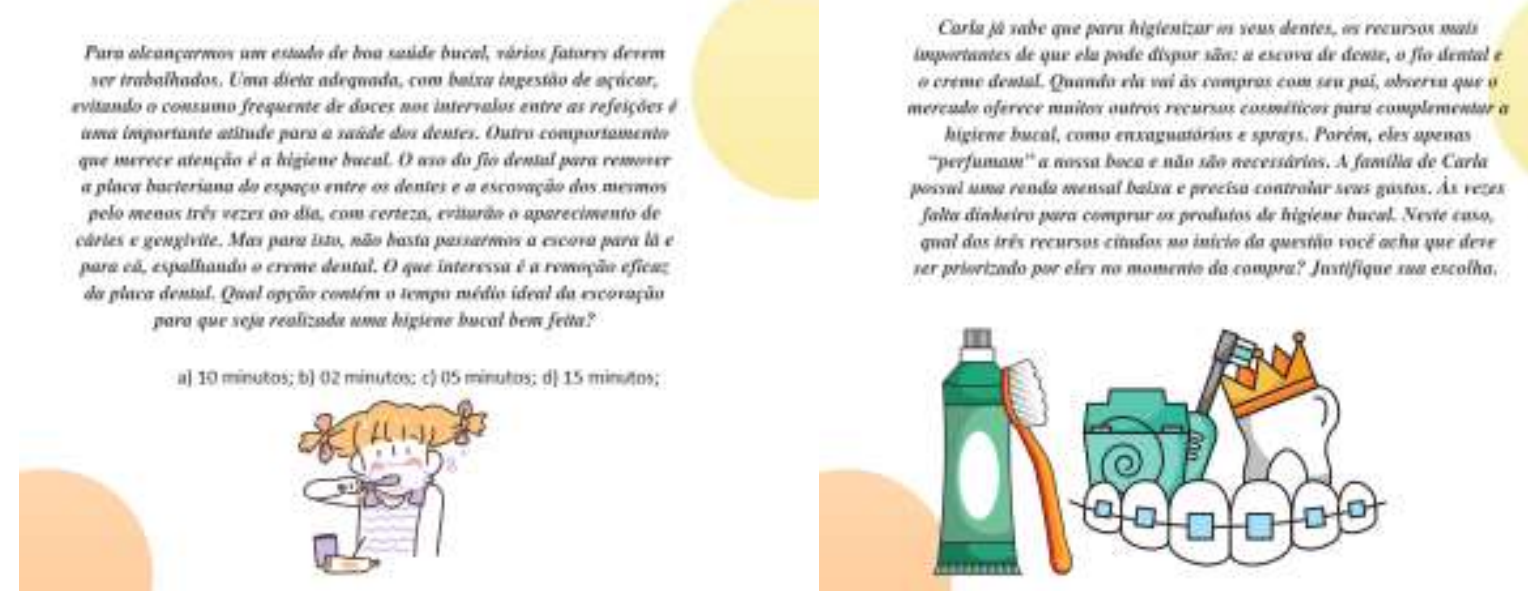

Fonte: Autores.

Bons hábitos de saúde bucal devem ser trabalhados desde cedo entre escolares. A conscientização sobre o uso de fio dental e escovação correta e frequente deve ser difundida também entre adolescentes e não somente entre as crianças. Mesmo que seja um tema repetitivo, deve ser repassado de forma dinâmica para que a aprendizagem seja significativa (Souza, 2017).

Para Pieri (2020), o avanço nas discussões relacionas à promoção em saúde dentro do ambiente escolar tem se intensificado nas últimas décadas, contudo, há a necessidade de se aplicar novas metodologias, como estratégias para repassar o conhecimento de forma mais dinâmica, colocando o ouvinte na posição de autor participativo e não somente de mero espectador. Para o desenvolvimento de tais ações, deve-se ainda, planejar intervenções de acordo com o contexto histórico social de cada cenário. 
Figura 3: Perguntas sobre bromidrose axilar e podal e cuidados com as fardas. Coelho Neto-MA, 2019.
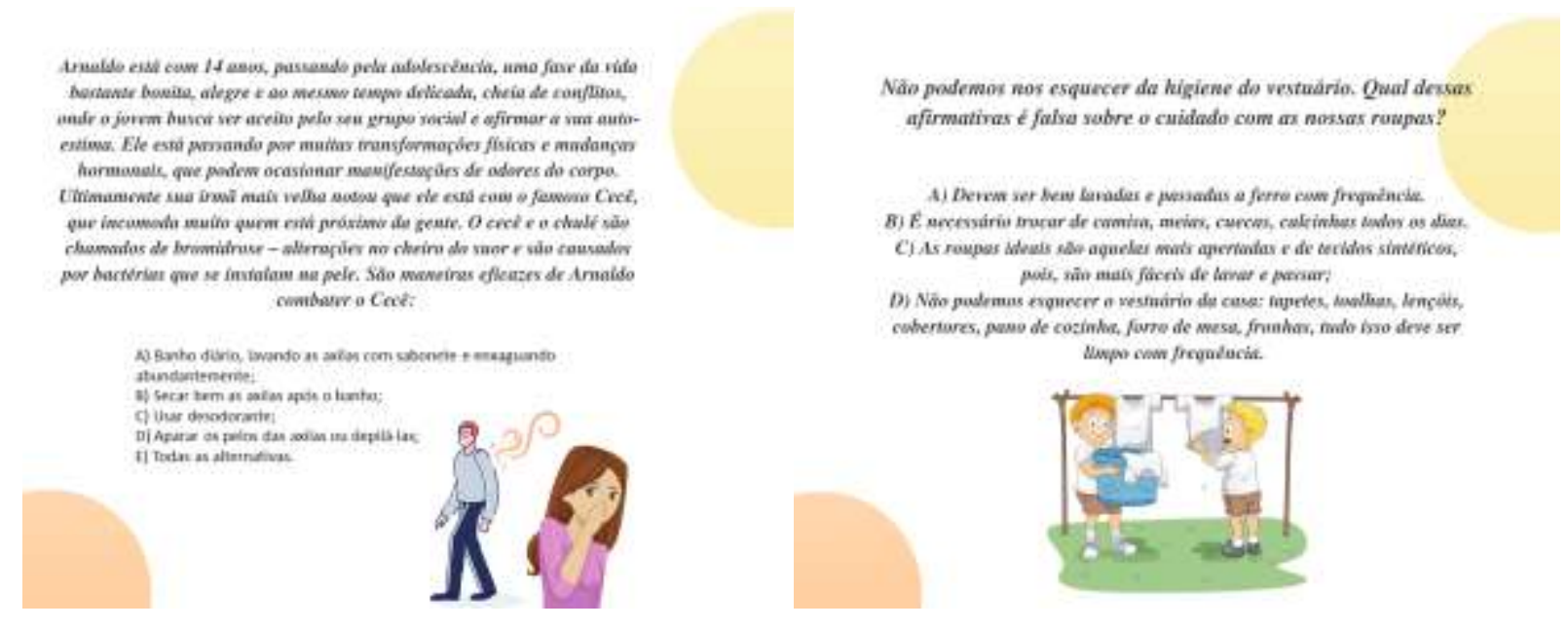

Fonte: Autores.

O mau cheiro axilar a plantar (chulé) foi observado entre os discentes. Percebeu-se também falta de higiene com relação ao fardamento, roupas sujas e com manchas na região axilar, sendo orientado a eles a importância em lavar a farda com frequência e não a usar durante a semana de aula sem fazer realizar a lavagem desta peça.

Foi explicado a eles que durante a adolescência as glândulas sudoríferas do corpo são mais ativas devido à puberdade e a produção do exsudado liberado pelas axilas, região íntima e pés podem apresentar odor fétido, causado pela decomposição do suor e debris celulares de bactérias e fungos e essa proliferação pode gerar infecções cutâneas (Santana, 2012).

Assim, foi orientado sobre as medidas que devem ser usadas para manter a higiene dessas regiões, como o uso de antissépticos em forma de pó, creme ou spray para controle dos fungos e bactérias ou caso não tenham condições para a compra de tais produtos, realizar a medida mais importante e que não tem nenhum custo, lavar e secar bem os pés, além de evitar sapatos quentes ou fechados por muito tempo, pois estes que induzam a transpiração dos pés (Filgueira, Araújo \& Silva, 2018).

Antes de cada pergunta, era realizada uma contextualização sobre o assunto ou o reato de um caso fictício para facilitar a resposta dos alunos (Figura 4). Infelizmente, muitos erravam questionamentos simples, constatando-se realmente, a falta de informação sobre higiene e confirmando as queixas dos professores a respeito do odor desagradável que permanecia nas aulas. 
Figura 4: Contextualização sobre higiene íntima e com os cabelos. Coelho Neto-MA, 2019.
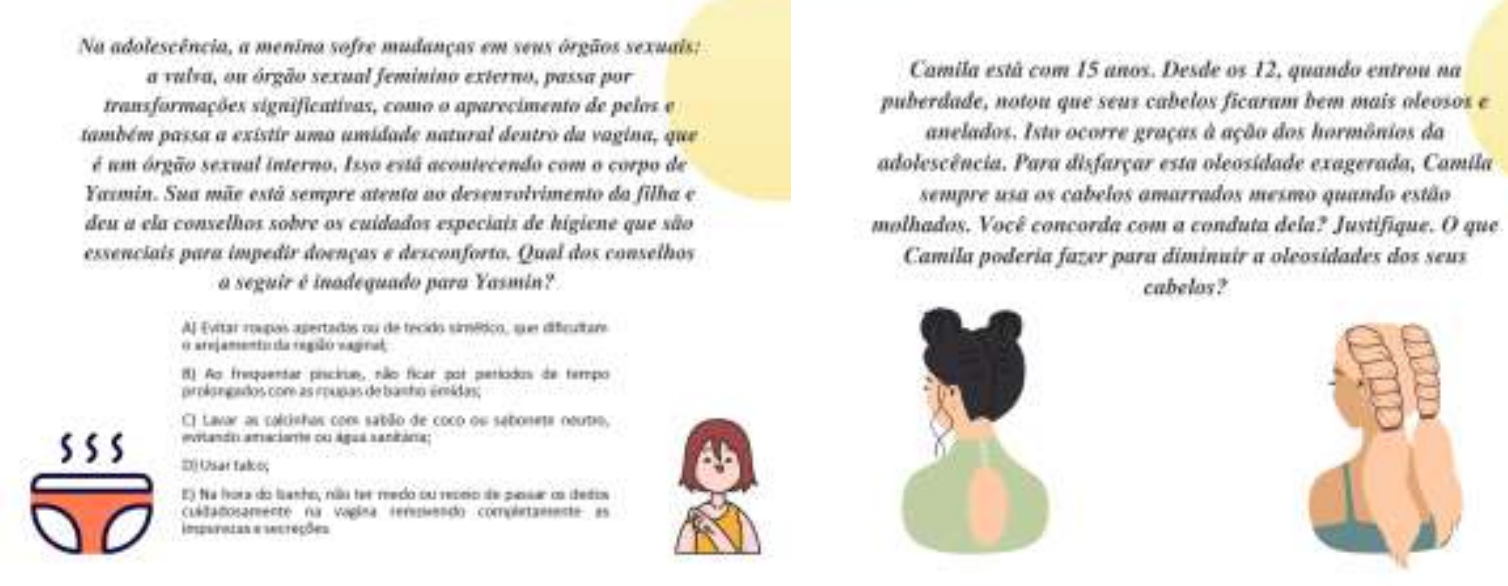

Fonte: Autores.

Após cada rodada, as condutoras faziam uma abordagem sobre o assunto de cada slide, repassando a resposta correta e aproveitando a oportunidade para trabalhar sobre o tema de forma leve e descontraída, repassando as informações necessárias para os discentes.

Para Copetti, Soares e Folmer (2018) uma aprendizagem baseada na contextualização está relacionada ao aprender e aprender a fazer, contribuindo para a formação do pensamento crítico. As autoras afirmam ainda que na educação em saúde dialógica, pautada na participação dos indivíduos, há melhores resultados, uma vez que os torna mais conscientes e responsáveis quanto a sua saúde pessoal e manutenção do que aprendeu.

A ideia de contextualização começou a ser defendida com a reforma do ensino médio, pela Lei de Diretrizes e Bases da Educação (LDB no 9.394/96), e é considerada como estratégia para vinculação do conhecimento com sua aplicação na prática (Pacheco, 2012).

Durante a infância e adolescência, o indivíduo adquire hábitos que podem ser mantidos durante a vida adulta, sendo de imprescindível o desenvolvimento de intervenções em saúde na escola para contribuir com a adoção de hábitos de higiene pessoal. Para atingir o objetivo, o processo educativo deve gerar autorreflexão e visão crítica (Martins, Horta \& Castro, 2013). A implantação da educação em saúde na escola facilita o levantamento de discussões e disseminação do conhecimento, visando a prevenção de agravos (Salum \& Monteiro, 2015; Santos, Teixeira \& Pereira, 2019).

Vale ressaltar que a ação desenvolvida tem trazido bons resultados, uma vez que é nítido a evolução dos discentes após o repasse das informações e orientações. Os alunos passam a ir pra escola com o fardamento limpo e com bons hábitos de higiene corporal, percebido e confirmado pelos próprios docentes. Nos anos posteriores, continuam com a postura e não há necessidade de intervenção das profissionais responsáveis pela atividade no segundo e terceiro anos.

\section{Considerações Finais}

A intervenção abordou temas necessários e poucos discutidos entre os adolescentes, na escola, como a higiene das mãos, unhas e cabelos e o risco de verminoses pela falta do cuidado. A higiene bucal também foi trabalhada, assim como os cuidados com o corpo e importância do banho, frisando sobre a doenças que podem ser geradas caso esses cuidados forem precários e quanto ao afastamento dos pares e colegas de sala, devido ao odor fétido do fardamento a mau hálito.

Assim, a elaboração da intervenção teve o intuito de fortalecer o conhecimento sobre higiene entre os discentes, visto que haviam muitas queixas da comunidade escolar, acerca da precariedade da higiene pessoal destes e a iniciativa da educação 
em saúde subsidia a construção de saberes com compromisso e responsabilidade social, uma vez que o incentivo do autocuidado deve estar atrelado a autonomia e protagonismo dos sujeitos, sendo necessário orientar e incentivá-los sobre a importância do tema trabalhado.

É importante salientar, que o objetivo do projeto foi alcançado e que após as intervenções realizadas, foi notável a melhora das condições de higiene entre os discentes, assim como em todos os anos em que a intervenção foi realizada. Assim, é de suma importância que sejam realizadas intervenções sobre a higiene pessoal de adolescentes escolares e que novas pesquisas venham agregar informações a fim de difundir o conhecimento sobre essa temática que é negligenciada nas escolas,

\section{Referências}

Bordenave, J. D. \& Pereira, A. M. Estratégias de ensino aprendizagem. (4a ed.), Vozes, 1989.

Cordeiro, N. V. (2019). Temas Contemporâneos e Transversais na BNCC: as contribuições da transdisciplinaridade. https://bdtd.ucb.br:8443/jspui/bitstream/tede/2661/2/NataliadeVasconcelosCordeiroDissertacao2019.pdf

Copetti, J., Soares, R. \& Folmer, V. (2018). Educação e saúde no contexto escolar: compartilhando vivências, explorando possibilidades. Uruguaiana, RS, Brasil. 183p.

Cordellini, J. V. F. (2016). Adolescência e a saúde física e mental. http://www.crianca.mppr.mp.br/modules/conteudo/conteudo.php?conteudo=444.

Cruz, J. D. S. (2018). Higiene pessoal como uma proposta inovadora para melhorar a qualidade de vida de crianças e adolescentes do centro de apoio lar peniel na cidade de simão dias-SE. $9^{\circ}$ Simpósio Internacional de Educação e Comunicação. Aracaju.

Filgueira, A. C. G, Araújo, G. M. S. \& Silva, J.V. (2018). Fique esperto;Orientações de saúde para adolescentes. organizadores Ana Cristina Gondim Filgueira, Gerliene Maria Silva Araújo, Janmille Valdivino da Silva; projeto gráfico, diagramação e capa Hanna Andreza Fernandes Sobral; revisão linguística Célio José Fiel da Silva Júnior. - Natal: IFRN. 230 p.

Guerin, C. S. et al. (2017). Promoting health education in a non-formal learning space. Revista Brasileira em Promoção da Saúde. $30(1): 5-12$.

Martins, A. S., Horta, N. C. \& Castro, M. C. G. (2013). Promoção da saúde do adolescente em ambiente escolar. Rev APS. 16(1): $112-116$.

Mori, M. S., Cabús, R. S. \& Freitas, S. R. S. (2016). Sequência didática sobre educação ambiental: uma abordagem metodológica alternativa para o ensino sobre a poluição atmosférica. Cadernos de Educação - reflexões e debates; 31 (15): 59-70.

Mouta, A. A. N. (2020). Saúde na escola: utilização do lúdico na educação básica para conscientização sobre a higienização pessoal e a prática da lavagem das mãos. REAS/EJCH, 1(50).

Pacheco, E. (2012). Perspectivas da educação Profissional técnica de nível médio Proposta de Diretrizes Curriculares Nacionais. São Paulo. 146p.

Pieri, A. S. (2020). Higiene E Saúde Na Escola. Revista de Formação e Prática Docente, (3):44-53.

Ramos, L. S. (2020). Instruções de higiene na escola e na sociedade como ação de saúde e prevenção de doenças: uma revisão bibliográfica. REAS/EJCH, 12(10).

Ruas, H. M. S. (2013). Desafio master de higiene na adolescência: um jogo didático para o ensino médio. 140p.

Salum, G. B \& Monteiro, L. S. (2015). Educação em saúde para adolescentes na escola: um relato de experiência. Revista Mineira de Enfermagem.19(2):246251.

Santos, T. B., Teixeira, C. \& Pereira, F. L. (2019). O projeto "Higiene e Saúde na Escola": reflexões sobre as estratégias de ensino e percepção dos conhecimentos relacionados à higiene e saúde entre estudantes de uma escola do campo. Interfaces - Revista de Extensão da UFMG, Belo Horizonte, 7(1):01591.

Santana, V. R. (2012). Perfil Demográfico De Crianças E Adolescentes Portadores De Hiperidrose Primária E A Avaliação Da Qualidade De Vida Após Simpatectomia Toracoscópica. Aracaju-SE.110p.

Silva, J. L. L. et al. (2020). Health education with teenagers ate school: experience report. Braz. J. Hea. Rev., 3(3), 6007-6017.

Souza, E. O. et al. (2017). Higiene bucal: Percepção dos alunos e modelo educativo. PECIBES, 2(56):56-63.

Terço, J. S. \& Freitas, S. R. S. 2016. Educação e saúde na escola: aplicação de uma sequência didática sobre alimentação saudável. Cadernos de Educação reflexões e debates.; 31 (15):71-85. 